\title{
Current Challenges in the Postoperative Management of Cataract Surgery
}

\author{
Alessandro Marchese and Francesco Bandello \\ Department of Ophthalmology, San Raffaele Scientific Institute, University Vita-Salute San Raffaele, Milan, Italy
}

DOI: https://doi.org/10.17925/OPHT.2021.15.1.15

\begin{abstract}
C ataract surgery has been the object of some great innovations in the last century, including advances in preoperative, intraoperative and postoperative management. Despite these advances, ophthalmologists still face many unmet needs in the care of patients requiring cataract surgery. Among the modern challenges of cataract surgery, the choice of antibiotic prophylaxis and antiinflammatory therapy are two important areas of interest that ensure a rapid recovery and help avoid severe postoperative complications. In this article, we review the current challenges of the postoperative management of cataract surgery, with insights into the options for preventing infections and inflammation after cataract surgery and directions for future research.
\end{abstract}

\section{Keywords}

Cataract, surgery, infection,

postoperative management

Disclosure: Francesco Bandello has received speaker's fees from the following: Allergan,

Alimera, Bayer, Farmila-Thea, Schering Pharma (S), Sanofi-Aventis, Novagali Pharma, Hoffmann-La Roche, Genetech, Novartis. Alessandro Marchese has no financial or non-financial relationships or activities to declare in relation to this article.

Review process: Double-blind peer review.

Compliance with ethics: This study involves a review of the literature and did not involve any studies with human or animal subjects performed by any of the authors.

Authorship: The named authors meet the International Committee of Medical Journal Editors (ICMJE) criteria for authorship of this manuscript, take responsibility for the integrity of the work as a whole, and have given final approval for the version to be published.

Access: This article is freely accessible at

touchOPHTHALMOLOGY.com (c) Touch Medical Media 2021.

\section{Received: 20 October 2020}

Accepted: 10 June 2021

Published online: 14 July 2021

Citation: touchREVIEWS in Ophthalmology. 2021;

15(1): 15-7

Corresponding author: Francesco Bandello,

Department of Ophthalmology, San Raffaele Scientific

Institute, University Vita-Salute San Raffaele, Via Olgettina

60, Milan, 20132, Italy. E: bandello.francesco@hsr.it

Support: No funding was received for

the publication of this article.
Cataract interventions are among the most common surgical procedures performed worldwide, in developed or developing countries. ${ }^{1}$ In 2015, approximately 3.6 million cataract interventions were performed in the United States. ${ }^{2}$ In the next few years, age-related diseases are likely to rise in many nations with increasing life expectancy, and cataracts will become more common.

Cataract surgery has always been at the centre of great innovation in the history of ophthalmology.-6 The three main areas of interest in cataract surgery regard the preoperative assessment, the surgical procedure and the postoperative management. Each of these equally contributes to the outcome, and has undergone significant innovation. $4,7,8$

A meticulous preoperative assessment not only ensures a safer procedure, but also sets the patient's expectations. ${ }^{9.10}$ Excessive expectations and growing pressure on surgeons are becoming more common in ophthalmology.10,11 Patients with ophthalmic pathologies, like advanced diabetic retinopathy, age-related macular degeneration, vitreoretinal diseases and uveitis, should be carefully informed about the expected outcomes before surgery and the risk of ocular complications. ${ }^{11,12}$ To assess the risks of suboptimal postoperative outcomes, optical coherence tomography has been introduced, with great benefit, in the preoperative assessment of patients. ${ }^{13}$ Also, a careful preoperative assessment is helpful in identifying high-risk patients, who may require specific preparations (e.g. preoperative topical non-steroidal anti-inflammatory drugs [NSAIDS] to help prevent miosis during surgery in specific cases).

The choice of intraocular lenses (IOLS) is another field that has seen great improvement. ${ }^{14}$ optical biometers are now available and have become more affordable, providing more accurate measurements than those provided by ultrasonography, and reaching optimal accuracy even in dense and advanced cataracts. ${ }^{15}$ Also, artificial intelligence is used to enhance the precision of IOL calculations and personalized approaches for both patients and surgeons. Postoperative adjustment of IOL power has recently become available with the introduction of novel IOL technologies. ${ }^{16} \mathrm{Also}$, progress in evaluating the anterior surface and the tear apparatus has shown that tear dysfunction can significantly affect surgical outcomes, including producing a less precise IOL calculation and a longer postoperative recovery. ${ }^{17}$

Many advances have also been made in the surgical procedure to achieve the optimal technique and level of experience. ${ }^{18}$ In most countries, phacoemulsification with IOL implant during primary surgery is the treatment of choice for cataract. However, other options are available where the access to phacoemulsification is more limited, including small incision cataract surgery, which represents a cost-effective and safe procedure for cataract removal. ${ }^{19}$ In developed countries, femtosecond laser-assisted cataract surgery is gaining popularity; however, this technique presents its own challenges. For instance, significant femto-induced preoperative miosis has been observed, though pre-treatment with NSAIDS seems to be protective..$^{20}$ Even if the procedure can be standardized, mastering these microsurgical techniques takes years of practice, and novice 
surgeons need to overcome significant difficulties to achieve a good surgical flow and meet the patient's expectations.

After the critical steps of preoperative examination and surgical intervention, postoperative management remains a crucial period in ensuring rapid and sustained recovery and healing, and preventing insidious complications. Different strategies have been adopted to ensure good postoperative outcomes, such as personalized approaches that account for multiple factors, including comorbidities and preoperative complications. Also, with the use of intraoperative antibiotics, the need for postoperative antibiotic prophylaxis has been questioned.

In this article, we review the current challenges and open questions in the postoperative management of cataract surgery, with insights into the current unmet needs and available treatment options.

\section{Unmet needs in the postoperative management of cataract surgery}

There are many unmet needs that ophthalmologists face in the postoperative care of patients undergoing cataract surgery. Most of these unmet needs derive from the inherent challenges of cataract surgery. Preventing infections and inflammation are two of the most crucial aims in the postoperative management of cataract surgery.

Postoperative infections have dramatic consequences and may lead to permanent vision loss. Given that the most common pathogens causing ocular infections after cataract surgery are bacteria, antibiotics are used intra- and postoperatively. At the end of the surgery, intracameral cefuroxime is now recommended in Europe. ${ }^{21}$ However, standardized protocols for the choice, duration and dose of postoperative antibiotic prophylaxis are still debated. A meta-analysis comparing the safety and efficacy of different intracameral antibiotics showed that cefuroxime and moxifloxacin reduced endophthalmitis rates. ${ }^{22}$ Also, intracameral antibiotics alone might be as effective as intracameral plus topical antibiotics, raising concerns regarding the need for postoperative prophylaxis, and supporting the possibility of dropless cataract surgery. ${ }^{22}$ Nevertheless, postoperative topical antibiotics are still broadly used. Shorter treatment regimens might reduce antibiotic resistance and induce better compliance, but the optimal treatment duration and compound are still debated.

Different classes of antibiotics are available in topical formulations for ophthalmic use, and combinations with corticosteroids have been suggested to increase treatment compliance. These combinations provide simultaneous anti-inflammatory and antibiotic prophylaxis after surgery. The choice of antibiotic after cataract surgery should consider the most frequent pathogens involved in postoperative endophthalmitis, which may vary across different locations. ${ }^{23}$ In Italy, the most diffuse bacteria causing postoperative endophthalmitis are coagulase-negative staphylococci, followed by Staphylococcus aureus, alpha-haemolytic streptococci, Pseudomonas and Streptococcus pneumoniae. ${ }^{23}$ In another study, the most common bacteria isolated from preoperative conjunctival flora included Staphylococcus aureus, Staphylococcus epidermidis and Gram-negative bacteria, including Pseudomonas aeruginosa. ${ }^{24}$ Among antibiotics, fluoroquinolones have a wide spectrum of action covering most of these pathogens (with the exception of coagulase-negative staphylococci); amphenicols (e.g. chloramphenicol) also cover most of these bacteria, excluding Pseudomonas; aminoglycosides (e.g. netilmicin) are also able to kill most of these bacteria (excluding streptococci). ${ }^{22}$ Various centres use combinations of two classes of these antibiotics due to their synergic actions, to provide comprehensive prophylaxis (e.g. chloramphenicol/netilmicin and fluoroquinolones). Also, regimens with a single antibiotic class are often used. For instance, previous studies have demonstrated the safety and efficacy of combination therapy with moxifloxacin and dexamethasone for 15 days to prevent infections and control inflammation after cataract surgery. ${ }^{25}$ However, despite fluoroquinolones being an effective choice, their prolonged use after uneventful cataract surgeries might come at the cost of antibiotic resistance. To address this issue, a recent international, multicentre, randomized, non-inferiority trial (EudraCT code: 2018-000286-36) in 808 patients tested the efficacy and safety of a 1-week course of topical levofloxacin plus dexamethasone eye drops after cataract surgery, followed by 1 week of dexamethasone alone. ${ }^{26}$ The main endpoint was the absence of inflammation in the anterior chamber 15 days after surgery as compared with a 2-week course of tobramycin plus dexamethasone; both the treatment groups received one drop four times daily. Both groups showed similar features at baseline, with a mean \pm standard deviation (SD) age of $71.98 \pm 8.59$ years and a mean \pm SD visual acuity of $0.41 \pm 0.25$ decimal. At the end of the treatment, $95.19 \%$ of the levofloxacin plus dexamethasone arm and $94.91 \%$ of the control arm had no inflammation in the anterior chamber (difference: 0.028; 95\% confidence interval: $-0.027,0.033$ ), demonstrating a non-inferiority of the short-course treatment with levofloxacin compared with the other regimen. ${ }^{26}$ Short antibiotic courses have advantages over longer therapies, such as fewer side effects, less bacterial resistance and, theoretically, lower treatment costs. ${ }^{23}$

Quick recovery after cataract surgery is expected by many patients. Surgical steps for cataract removal and IOL placement are at risk of postoperative inflammation. Corneal oedema represents one of the side effects of significant concern, especially in the first weeks following surgery. In the non-inferiority trial mentioned above, corneal oedema was observed at similar rates in patients treated with the levofloxacin and dexamethasone treatment regimen compared with the tobramycin plus dexamethasone regimen (3.29\% and $4.83 \%$ of patients, respectively). ${ }^{26}$ Touching or damaging the iris increases the risk of ocular inflammation, not only in the anterior segment, but also in the retina, with macular oedema. More than $95 \%$ of the patients treated with levofloxacin and dexamethasone had no ocular inflammation 15 days after cataract surgery, demonstrating that this postoperative management may help to achieve the goal of rapid and safe recovery. ${ }^{26}$ Long-term postoperative management after cataract surgery is another topic of debate. Additional studies are awaited regarding the optimal duration and choice of NSAIDS, the use of lubricants and the timing of follow-up.

Overall, a shorter antibiotic course after cataract surgery seems to provide some advantages over standard therapies in maintaining a good safety profile for patients. In particular, the reduced risk of creating antibiotic resistance to the most potent classes of topical antibiotics available for ophthalmic use is of great benefit to both patients and care providers. Future studies should address whether these advantages are confirmed over longer follow-up and if these regimens provide different results compared with other postoperative regimens and dropless cataract surgery.

\section{Conclusions}

Cataract surgery still poses many challenges, despite many improvements in preoperative, preoperative and postoperative management. Given the high number of patients requiring cataract surgery, even minor adjustments to therapy may achieve great benefits for the ophthalmic 
community and patients by reducing costs, side effects and antibiotic resistance. The optimal treatment strategies after cataract surgery implement the most recent evidence in clinical practice, which often comes from multicentre, randomized clinical trials. Shorter antibiotic courses with broad-spectrum antibiotics in the postoperative care of patients after cataract surgery, should be considered and further studied. While the ultimate goal of ophthalmic surgery remains that of achieving the maximal vision for each patient, preserving our armamentarium to fight ocular infections should be a key strategy in ensuring optimal visual outcomes for the highest number of patients. $\square$
1. Cataract surgical rates. Community Eye Health. 2017;30:88-9.

2. Grzybowski A. Recent developments in cataract surgery. Ann Trans/ Med. 2020; 8:1540.

3. Fontana $\mathrm{L}$, Coassin $\mathrm{M}$, lovieno $\mathrm{A}$, et al. Cataract surgery in patients with pseudoex-foliation syndrome: current updates. Clin Ophthalmol. 2017;11:1377-83.

4. Nagy ZZ, MCAlinden C. Femtosecond laser cataract surgery. Eye Vis (Lond). 2015;2:11.

5. Little BC, Smith JH, Packer M. Little capsulorhexis tear-out rescue. J Cataract Refract Surg. 2006;32:1420-2.

6. Sharma B, Abell RG, Arora T, et al. Techniques of anterio capsulotomy in cataract surgery. Indian J Ophthalmo. 2019;67:450-60

7. Savini G, Hoffer KJ. Intraocular lens power calculation in eyes with previous corneal refractive surgery. Eye Vis (Lond). 2018:5:18.

8. Wielders LHP, Schouten J, Winkens B, et al. European multicenter trial of the prevention of cystoid macular edema after cataract surgery in nondiabetics: ESCRS PREMED study report 1. J Cataract Refract Surg. 2018:44:429-39.

9. Wisely $\mathrm{CE}$, Robbins $\mathrm{CB}$, Stinnett $\mathrm{S}$, et al. Impact of preoperative video education for cataract surgery on patient learning outcomes. Clin Ophthalmol. 2020:14:1365-71.

10. Chen Z, Lin X, Qu B, et al. Preoperative expectations and postoperative outcomes of visual functioning among cataract patients in urban southern china PLOS One 2017:12:e0169844. 11. Chan NS, Ti SE, Chee SP. Decision-making and management of uveitic cataract. Indian J Ophthalmol. 2017;65:1329-39.

12. Hardin JS, Gauldin DW, Soliman MK, et al. Cataract surgen outcomes in eyes with primary epiretinal membrane. JAMA Ophthalmol. 2018;136:148-54.

13. Kowallick A, Fischer CV, Hoerauf H. Optical coherence tomography findings in patients prior to cataract surgery regarded as unremarkable with ophthalmoscopy. PLOS One 2018:13:e0208980

14. Xia T, Martinez CE, Tsai LM. Update on intraocular lens formulas and calculations. Asia Pac J Ophthalmol (Phila). 2020;9:186-93.

15. An Y, Kang EK, Kim H, et al. Accuracy of swept-source optical coherence tomography based biometry for intraocular lens power calculation: a retrospective cross-sectional study. BMC Ophthalmol. 2019;19:30.

16. Dick HB, Gerste RD. Future intraocular lens technologies. Ophthalmology. 2020; S0161-6420(20)31202-1. [Online ahead of print].

17. Labetoulle M, Rousseau A, Baudouin C. Management of dry eye disease to optimize cataract surgery outcomes: two tables for a daily clinical practice. J Fr Ophtalmol. 2019;42:907-12.

18. Coppola M, Marchese A, Rabiolo A, et al. Comparison of two popular nuclear disassembly techniques for cataract surgeons popular nuclear disassembly techniques for cataract surg
in training: divide and conquer versus stop and chop. Int Ophthalmol. 2019:39:2097-102.

19. Dean WH. Quality of small incision cataract surgery. Community Eye Health. 2015:28:30-1.

20. Diakonis VF, Kontadakis GA, Anagnostopoulos AG, et al. Effects of short-term preoperative topical ketorolac on pupil diameter in eyes undergoing femtosecond laser-assisted capsulotomy. $\rfloor$ Refract Surg. 2017;33:230-4.

21. Barry P. Adoption of intracameral antibiotic prophylaxis of endophthalmitis following cataract surgery: update on the escrs endophthalmitis study I Cotaract Refract Surg. 2014;40:138-42.

22. Bowen RC Zhou AX Bondalapati S et al Comparative analysis of the safety and efficacy of intracameral cefuroxime moxifloxacin and vancomycin at the end of cataract surgery: a meta-analysis. Br J Ophthalmol. 2018:102:1268-76.

23. Grosso A, Ceruti P, Scarpa G, et al. Choosing wisely and the use of antibiotics in ophthalmic surgery: There is more than meets the eye. Eur J Ophthalmol. 2018;28:625-32.

24. Bruttini C, Pallone C, Verticchio Vercellin A, et al. Pre-operative conjunctival flora in patients with local and/or systemic risk factors for post cataract surgery infection in northern italy. Eur Ophthalmol. 2020:1120672120934991.

25. Freitas LL, Soriano E, Muccioli C, et al. Efficacy and tolerability of a combined moxifloxacin/dexamethasone formulation for topical prophylaxis and reduction of inflammation in phacoemulsification: a comparative, double masked clinical trial. Curr Med Res Opin. 2007:23:3123-30.

26. Bandello F Coassin M, Di Zazzo A et al. One week of levofloxacin plus dexamethasone eye drops for cataract surgery: an innovative and rational therapeutic strategy. Eye (Lond). 2020;34:2112-22. 